SIALOLITHIASIS AND SIALODOCHITIS IN CHILDHOOD *

\author{
HAROLD NEUHOF, M.D. \\ NEW YORK
}

During the time that observations were made on a recently reported variety of sublingual gland inflammation ${ }^{1}$ notes were kept of other affections of the salivary glands seen in children. Three cases of salivary calculus and two of chronic sialodochitis were encountered. ${ }^{2}$

In his classical work on salivary calculus, published in 1855, Thomas de Closmadeuc stated that sialolithiasis did not exist in children; its occurrence in an individual of 20 years was the youngest case recorded at that time. Since then, however, seven cases of salivary calculus in children have been reported. ${ }^{3}$ In adding those I have observed, I wish to show that sialolithiasis is not an exceedingly rare condition in children, and to depict its symptomatology in early life.

Chronic inflammation and stenosis of the salivary duct, in the form observed by me in two cases, does not appear to have been described. The affection is of some importance; in both cases the diagnosis of parotid neoplasm had been made and excision proposed. Sialodochitis bears no demonstrable relationship to salivary calculus; it is convenient, however, to discuss both affections in one paper.

For purposes of comparison, the manifestations of sialolithiasis in adults will be considered very briefly. Its frequency in adult life is suggested by some 400 cases that have been described, groups of eight or more often being reported by individual observers. Calculi are encountered most frequently in the excretory ducts; in Wharton's duct in the great majority of instances. The submaxillary gland, likewise, is the most common seat of the gland stones. A distinction between duct and gland concretions is made because it is as yet impossible to determine if duct stones are extruded gland stones, or whether the conditions are separate ones. With the calculus in either situation, inflammatory and sclerotic changes in the gland ensue.

Most observers now agree that sialolithiasis results primarily from bacterial invasion; some, however, presume that swelling of the duct, with retention and inspissation of saliva, is the primary cause, and the

* Submitted for publication Jan. 6, 1916.

1. Neuhof, H. : Am. Jour. Dis. Child., August, 1915, p. 94.

2. With one exception, all were seen and treated in the morning surgical clinic of Mount Sinai Hospital.

3. The reports of only three were found in a careful search of the literature. The remaining four are quoted by various authors, either without reference to their source or with references that were inaccessible or incorrect. 
bacterial element secondary. In fact, the etiologic factors are ill understood at present. For example, there is no adequate explanation for the far greater incidence of sialolithiasis in the male sex, or of the preponderence of calculi in the submaxillary duct-gland system, of recurrences after removal, etc.

The symptoms of duct stones are usually more severe and well defined than those of gland stones; calculi in Wharton's duct generally result in the most pronounced manifestations. Wherever the stone is situated there are usually two periods in the symptomatology. The first is characterized by intermittent attacks of pain in the affected side of the mouth, sometimes severe enough to be dignified by the term "salivary colic." The coincident appearance of a sensitive salivary swelling is very characteristic; the mass, tense and firm, is generally in the immediate region of the calculus. Suppuration marks the second stage. Mucopurulent discharge, of varying amounts, escapes from the orifice of the duct, the latter then becoming reddened and swollen. Abscesses about the gland or duct may develop, to burrow externally or ultimately to rupture into the mouth.

A calculus situated near the orifice of a duct is readily palpable through the mucous membrane of the mouth in most instances; in the depths of the duct it is frequently but by no means invariably felt by probing the canal. The diagnosis of a gland stone, on the other hand, is chiefly based on the indirect evidence of the history and the clinical findings; Roentgen-ray examination has revealed calculi in the gland in a few instances.

Many less important symptoms and physical signs have been omitted; enough has been categorically stated to indicate that the diagnosis of salivary calculus would not be difficult if the cardinal manifestations were present regularly. On the contrary, however, the symptoms are often bizarre; acute attacks of sialolithiasis have been mistaken for alveolar periostitis, cervical adenitis, angina Ludovici, etc., and the condition, in a chronic form, has been confused with tuberculosis, gumma, and malignant disease of the salivary gland.

\section{SIALOLITHIASIS IN CHILDREN}

In the reported cases, and in two of the three I have observed, the symptoms were neither as severe as those generally seen in adults nor of as long duration before the patients came under observation. Of my three cases, two were instances of solitary calculus in Wharton's duct, the third had multiple calculi in Stenson's duct. The latter, of unusual interest, will be described first.

CAse 1.-A girl, 6 $1 / 2$ years old, came under observation in the Mount Sinai Hospital Surgical Clinic in July, 1915 (transferred to me through the courtesy of Dr. S. Cohn). She had had measles and summer diarrhea in infancy. 
When she was $2 \frac{1}{2}$ years old both ear drums were punctured for an acute inflammation, following grip (?). A swelling of the right side of the face developed soon after; this was termed mumps. The tumefaction, always sensitive and occasionally very painful, has never disappeared since that time. It has been of fairly constant size in summer, decidedly larger and with greater fluctuations in its proportions in the winter. Three or four times in the latter season very acute swelling, with fever, would develop, persist for two or three days, and then slowly recede.

Nine months before the patient was first seen, discharge of pus from the mouth was noted. It has continued ever since. Although usually inconsiderable, thick, and ropy, on a few occasions the discharge has been very profuse and thin. The swelling in the right cheek has been less marked and less painful since the onset of the discharge.

Examination.-The child is poorly nourished. General examination is negative. Teeth and buccal mucous membrane are normal. There is a very pronounced tumefaction of the right side of the face, outlining the enlarged parotid gland. On palpation, the gland is very firm and moderately tender; there are a few softer areas in the indurated mass. The orifice of Stenson's duct is very small and reddened, contrasting with the larger, normal orifice on the left side. By pressure on the parotid mass a little saliva mixed with considerable pus is expressed from the duct. The finest lacrymal probe could not be passed into the duct. Roentgen-ray examination was negative. In order to clear up the diagnosis it was determined to split the orifice of the duct and explore its contents.

Operation.--Under general anesthesia, the mouth of the duct was widely incised, thick mucopurulent fluid escaping. A good sized probe could then be passed into the duct. At a depth of four $\mathrm{cm}$. a calculus was encountered. After some manipulations the probe could be carried past the calculus to the junction of the duct with the gland. Here a second calculus was felt. Ineffectual attempts were made to extract the stones from the duct.

Course.-The parotid swelling began to diminish soon after this operation and the patient felt better. In two weeks the tumefaction had almost disappeared. The discharge of pus steadily diminished, the proportion of saliva increasing with the reduction of the swelling. The calculi, now close together, could easily be felt through the cheek and the mouth, as well as by probing the duct.

One month later the parotid swelling was practically gone, the discharge of pus had ceased, and the child was quite well except for the presence of the calculi in the duct. There was no tendency toward closure of the slit orifice. Another ineffectual effort was made to extract the stones from the duct. Evidently they lay beyond a stricture of the canal, and therefore could not be grasped and removed. An open operation for their removal was refused. When recently seen (October, 1915) the patient was still quite well. The swelling of the parotid was gone. The calculi were easily felt through the cheek.

CASE 2.-A boy, 5 years old, came under observation in the surgical clinic of Mt. Sinai Hospital in August, 1911, with a two months' complaint of pain under the tongue and along the floor of the mouth. Pain had been continuous, not severe, and not progressive. Other symptoms had not been noted. There were no previous illnesses other than measles, and nothing in the history of the case to throw any light on the cause of the present affection.

Examination.-The general physical examination was negative. The right submaxillary gland was slightly enlarged and sensitive. To the right of the frenum linguae the mucous membrane and submucous tissues of the floor of the mouth were somewhat edematous. In this area a small, firm, rounded mass (the calculus) was indefinitely felt. The orifice of Wharton's duct appeared unchanged, but a fine probe could not be introduced. Saliva could not be expressed from the duct. 
Treatment and Course.-Under local anesthesia a small incision was made through the duct over the calculus; the latter was then readily extracted. Escape of thick saliva ensued. The stone was somewhat larger than a green pea, egg shaped (the small end pointing to the orifice of the duct), and of slightly irregular surface. On chemical examination (chemical laboratory, Dr. Bookman) it was found to consist entirely of calcium phosphate.

The postoperative course was uneventful. The discharge of saliva continued through the new opening of Wharton's duct. The swelling of the submaxillary gland disappeared. The remainder of the duct was probed on several occasions and found free. About two months after operation the patient disappeared from observation.

CASE 3.-A boy, 8 years old, came to the morning surgical clinic of Mt. Sinai Hospital in May, 1912, complaining of a sense of discomfort in the floor of the mouth, of one week's duration. There was no similar previous complaint. Scarlet and diphtheria when $6 \frac{1}{2}$ years old. There was no history of any mouth infection or injury.

Examination.-The general examination of the robust patient was negative. On the right side of the floor of the mouth, close to the orifice of Wharton's duct, a calculus could be easily felt. The overlying mucous membrane was unchanged. Saliva was not seen escaping from the unaltered orifice of Wharton's duct. The submaxillary gland was not palpable.

Treatment.-Under local anesthesia an incision was made over the calculus and was extended through the mouth of the duct. The calculus was extruded from the incision by the dammed-up saliva, which then escaped in considerable quantities. The dilated duct was probed to the gland and was found free. The calculus was slightly smaller than a lima bean, of spheroidal shape, very light and fragile, and of a spongy appearance; unfortunately it was misplaced and was not examined. The patient did not return for further treatment.

The cases of salivary calculus in childhood, whose reports in the literature are accessible, are as follows:

CASE 4. - The patient, the youngest on record, was a male infant, 3 weeks old. The mother took the child to the physicians because it nursed with great difficulty. The tongue was found markedly displaced by a large, soft, sublingual swelling, presenting a firmer area in the center. A diagnosis of ranula was made. On pressing the mass, however, the tip of a calculus was seen protruding from the orifice of the duct. After several attempts the calculus was finally removed without an incision. The child remained well. The calculus was the size and shape of a grain of wheat, with one sharply pointed extremity, of yellowish color, and granulated surface. It was composed of phosphate of lime and mucus. The authors believed that it developed in fetal life.

CASE 5. - Girl, 12 years old. There was a three months' history of intermittent swelling in the sublingual region. Each recession of the swelling was accompanied by a profuse discharge of saliva and a very small amount of pus. The patient complained of some interference with talking and swallowing. On examination, a calculus was found engaged in Wharton's duct. It could be rolled under the finger. About the calculus there was a soft swelling, the size of a "nut." The duct over the swelling was incised and the calculus removed. Its extraction was followed by the escape of considerable saliva, and the disappearance of the mass. Cultures from the center of the mass were sterile.

CASE 6. ${ }^{\circ}-$ A boy, 13 years old. For three years there had been pain in the right side of the mouth during mastication, pain in the right cheek, and inter-

4. Burdel and Cloquet: Comp. rend. Acad. de sc., No. 50, p. 893.

5. Péraire and Gaudier: Bull. Soc. anat. de Paris, 1898.

6. Solé : Presse méd. Belge, 1897, xlix, 113. 
mittent swelling of the cheek in cold and damp weather. These symptoms were gradually progressive. On examination, the right cheek was found slightly swollen, the venules of the overlying skin slightly dilated. Palpation revealed a series of nodules in the course of Stenson's duct, more readily felt when the masseters were contracted. By probing, the duct was found abnormally arched. Six calculi were extracted, through an external incision of the duct. They varied in weight from 0.006 to $0.170 \mathrm{gm}$. The incision in the duct was sutured with catgut; the postoperative course was smooth and there was no leakage of saliva from the wound.

The remaining cases, whose reports are not accessible are: Two cases presumably reported by Schenk, one in a child 7 years old, the other in a child of 12 . Wright (in a monograph on the Physiology and Pathology of the Saliva, 1892) is said to have described a salivary calculus in a child 9 years old. Reboul's ${ }^{7}$ case occurred in a child 9 (or 15 ) years old. Beyond the bare statement of the existence of salivary stone in these children the authors referring to them go no further; there are no descriptions of clinical findings or of the results of treatment.

\section{SIALODOCHITIS IN CHILDREN}

Two varieties of chronic sialodochitis have been described. They are affections of adult life; in a very few instances, however, their onset in childhood has been observed. Although they bear no relation to the type of sialodochitis I wish to describe, a short presentation of the clinical pictures may be permitted.

One variety-the sialodochitis fibrinosa of Kussmaul-is characterized by sudden and recurrent attacks of duct obstruction caused by fibrin plugs. This very rare affection is exceedingly chronic, affects Stenson's ducts in most instances, and is generally bilateral. The earliest manifestations of some of these cases date from childhood. The second type is, practically, identical in the recurrence of attacks, in its chronicity, and the almost exclusive, generally bilateral involvement of Stenson's ducts; it is chiefly differentiated by the absence of duct plugs. One case has been observed in childhood: ${ }^{8} \mathrm{~A}$ boy 13 years old suffered from intermittent attacks of pain and parotid swelling for about one year. The duct was dilated, considerable pus escaped from its mouth and the entire course of the duct could be readily probed.

These peculiar, very obscure (and probably constitutional) affections are the only forms of chronic sialodochitis that appear to have been recorded, either in adult life or in childhood. The type $I$ wish to describe is characterized by a cicatricial stenosis of one Stenson's duct (the opposite one being normal), the result of an inflammation of unknown origin, by a firm, nodular enlargement of the corresponding

7. Said to have been reported in the Echo méd des Cévennes, 1902.

8. Noeggerrath: Charité Annalen, 1909, p. 176. 
parotid gland, by a tendency to recurrence after slitting the mouth of the duct, but ultimate cure after the stenosis has been permanently overcome.

CASE 1.-A boy 4 years old came under observation in the morning surgical clinic of Mount Sinai Hospital in September, 1913, through the courtesy of Dr. Stephen Greenfield. There was an enlargement in the right parotid region, of five months' duration. The mass had slowly increased in size, pain in and about it had been dull and constant, some loss in weight had been noted. There were no previous illnesses other than measles at the age of 2 years, and pertussis soon after. A diagnosis of sarcoma of the parotid gland had been made and excision advised.

Physical Examination.-A poorly nourished pale child. The general examination is negative. The buccal mucous membrane is normal. The left parotid gland and duct reveal no abnormalities. The right parotid gland is enlarged to about three times the normal size; is firm throughout, but with more solid, irregular, pea-sized nodules scattered near the surface and in the depths. The mass is moderately sensitive to pressure. It is fixed to the underlying tissues. There are a few enlarged, tender lymphnodes in the right submaxillary triangle. The parotid duct is felt as a firm, rigid strand in the cheek. The mouth of the duct can be barely distinguished. It makes a tiny pale depression in the buccal mucous membrane; saliva does not escape when the parotid mass is compressed. The finest lacrymal probe cannot be introduced into the orifice of the duct.

The diagnosis of parotid sarcoma would have been concurred in were it not for the duct findings. The parotid swelling itself appeared absolutely typical. Slitting of the duct (in order to explore it) was suggested before sending the case to the wards.

Treatment and Course-General anesthesia. The smallest probe could be passed into the very narrow, stiff-walled duct for about $1 \mathrm{~cm}$. A tight stenosis was then met. With a little manipulation the probe was pushed through a stricture less than $1 \mathrm{~cm}$. long and was then passed into the dilated duct beyond. The cicatricially stenosed part of the duct was slit open from the orifice; the result was a very wide opening leading to the dilated duct. Quantities of thick mucoid saliva escaped. The lining of the dilated part of the duct was then seen to be much thickened, congested, and edematous. A large probe could be introduced to the gland. It described a somewhat irregular course but met no other. strictures. The wall of the duct felt very rigid.

Two weeks after operation the parotid tumor had diminished greatly in size and was much softer and more homogeneous in consistency. The only postoperative treatment was pressure made over the gland, at each visit to the clinic (resulting in profuse discharge of saliva from the duct). No visible swelling remained at the end of one month, but the gland was, as yet, firmer and more resistant to touch than the normal. The child disappeared from observation at that time, to return five weeks later with a recurrence of the parotid swelling (to about half the size of the original tumor) and of the stenosis of the duct orifice. The latter was again slit open under general anesthesia; the gland swelling promptly receded. Progressive dilatation of the buccal end of the duct was then practiced for several weeks. Instructions were given for the return of the child twice a month, for the passage of sounds. This was not done, and the patient was not seen again until two and a half months later. The duct mouth was then found partly closed and the parotid gland slightly enlarged. The duct was slit a third time, and there was the same prompt reduction in the size of the parotid. With dilatation by probes for a few weeks the duct has remained patent permanently. When last seen, in July, 1915 (about two years after operation), the patient was well and in excellent general condition. The orifice of the duct was slightly larger than 
the normal, could be probed easily; normal-looking saliva escaped; no enlargement of the parotid gland was discernible.

CASE 2.-A girl 8 years old came under my observation in May, 1915, with a swelling in the right parotid region, of four months' duration. The mass increased in size rapidly in the first few weeks, more slowly thereafter. In the first two weeks there was slight fever, and considerable pain in the cheek. The latter was largely controlled by the application of hot poultices. Changes in secretion of saliva were not noted. The physician in charge stated that the parotid swelling was uniform at first, in every way identical with mumps; subsequently, however, the mass became irregularly nodular. There was never any discharge of pus into the mouth. The diagnosis of "mixed tumor" of the parotid had been made, and excision advised. The only suggestive feature in the previous history was that extensive wiring of the teeth to adjust the "bite" had been completed about two months before the onset of the parotid swelling.

The general examination was negative. There was no existing lesion and no evidence of a previous one in the buccal mucous membrane. The left parotid gland and duct were normal. The mucous membrane immediately around the orifice of the right duct was pale and firm to touch. The mouth of the duct was exceedingly small and evidently stenosed. The finest lacrymal probe was introduced with some difficulty. It traversed a very narrow, irregular course for some $2 \mathrm{~cm}$., then suddenly entered the (apparently) dilated duct beyond, and went directly to the gland. The parotid gland was about the size and shape of an English walnut, slightly sensitive, rather firm and resistant to touch, with a number of shotty nodules scattered near the surface and in the depths. The mass was slightly moveable on the underlying tissues. Without the presence of the sound the course of the duct in the cheek could not be felt. (Compare with Case 1.)

The diagnosis of sialodochitis with duct stenosis was made, on the basis of the experiences in the former case. In order to avoid the postoperative recurrences of the stenosis excision of the sclerotic part of the duct was planned.

Treatment and Course.-Under local anesthesia the cicatricial tissue around the mouth of the duct was freed. With a probe in the duct as a guide the distal, stenosed end of the canal was isolated and removed with the tissue about the duct orifice. Immediately following the excision there was a profuse flow of glairy saliva mixed with thin watery fluid (cultures sterile). The remaining part of the duct could be probed readily, felt stiff and resistant, and was evidently dilated. A single cat-gut suture approximated the new orifice of the duct to the mucous membrane. Two sutures closed the remainder of the wound to within a short distance of that orifice.

The postoperative course was smooth. Saliva discharged freely from the new duct mouth, the parotid swelling steadily decreased. In three weeks the gland was of almost normal size and much softer in consistency; the nodules were also much softer as well as more indefinite. Two months after operation the gland was barely palpable, the nodules had disappeared, the discharge of saliva was free, the entire course of the duct could be readily probed, and there was no tendency to recurrence of stenosis. When seen recently (November, 1915, six months after operation) the child was quite well; a difference between the parotid glands was not discernible, the new duct orifice remained patent, probing it revealed no abnormalities.

\section{CONCLUSIONS}

1. Sialolithiasis in childhood cannot be termed the exceedingly rare, almost unknown condition it is presumed to be. The manifestations are more clean-cut and evident in children than in adults, the diagnosis 
can be made more readily, the surgical treatment is simple and efficacious. The salivary duct should be probed in every instance of enlargement of a salivary gland in a child when a definite cause for the enlargement cannot be ascertained.

2. There is a hitherto undescribed form of sialodochitis of Stenson's duct in children, secondary to inflammation of unknown origin, leading to an enlargement of the parotid gland that can be readily mistaken for sarcoma or mixed tumor. The gland is considerably increased in size, firm, nodular, adherent; the orifice and buccal end of the duct are embedded in stenosing cicatricial tissue. There is a tendency to repeated recurrences of the parotid swelling after slitting the mouth of the duct, but cure follows promptly the excision of the diseased end of the duct.

1275 Madison Avenue. 